\title{
卟啉-二芳基乙烯分子及其金属配合物的合成与光致变色特性
}

\author{
郑春梅王婷婷 曾和平* \\ (华南理工大学化学与化工学院功能分子研究所 广州 510640)
}

\begin{abstract}
摘要 以苯甲醛和吡咯为初始原料, 经多步反应合成了 5-[2,3-双 (2,4,5-三甲基-3-噻吩基)马来酰亚胺- $N$-苯基]-10,15,20三苯基卟啉(TPPMA)及其金属锌配合物(ZnTPPMA)与铜配合物(CuTPPMA), 通过 IR, MS, ${ }^{1} \mathrm{H}$ NMR 和 ${ }^{13} \mathrm{C}$ NMR 确认了 化合物的结构, 并利用 UV-Vis 光谱探讨了化合物的光致变色性能. 实验结果表明, 无论在聚乙烯醇缩丁醛(PVB)膜还 是在溶液中, TPPMA 在 $254 \mathrm{~nm}$ 紫外光照射下, 没有光致变色现象, 而在 254 和 $650 \mathrm{~nm}$ 的光照下其金属配合物 CuTPPMA 和 ZnTPPMA 在溶液及 PVB 膜中皆可以发生可逆的光致变色反应.
\end{abstract}

关键词 光致变色; 卟啉一二芳基乙烯; 金属配合物

\section{Synthesis and Photochromic Properties of Diarylethene-Based Porphyrin Derivative and Its Metal Complexes}

\author{
Zheng, Chunmei Wang, Tingting Zeng, Heping* \\ (Institute of Functional Molecules, School of Chemistry and Chemical Engineering, South China University of Technology, \\ Guangzhou 510641)
}

\begin{abstract}
Bis(2,4,5-trimethyl-3-thienyl)maleimide- $N$-phenyl]-10,15,20-triphenylorphyrin (TPPMA) and its $\mathrm{Zn} / \mathrm{Cu}$ complexes have been synthesized using benzaldehyde and pyrrole as starting materials. Their structures were confirmed by ${ }^{1} \mathrm{H}$ NMR and ${ }^{13} \mathrm{C}$ NMR, IR, MS techniques. The photochromic properties were determined by UV-Vis spectra. The results indicated that TPPMA was non-photochromic under $254 \mathrm{~nm}$ light either in solid or liquid phase, while ZnTPPMA and CuTPPMA underwent reversible photochromic reactions under 254 and $650 \mathrm{~nm}$ light both in solution and in PVB amorphous film.

Keywords photochromic; porphyrin-diarylethene; metal complexes
\end{abstract}

二芳基乙烯类光致变色分子由于其良好的热稳定 性、耐疲劳性、高的量子产率及快的响应时间等优 点 $^{[1 \sim 5]}$, 引起人们的广泛关注 ${ }^{[6-8]} .1988$ 年, Irie 等 ${ }^{[9]}$ 首次 将 1,2-二氧基-1,2-双(2,4,5-三甲基-3-噻吩)乙烯水解得到 2,3-双(2,4,5-三甲基-3-噻吩)马来酸䣶(BTE), 它可以在 $\lambda=405$ 及 $>520 \mathrm{~nm}$ 光照条件下发生可逆的开关环反应. 在固态时, BTE 的胶体溶液、多晶都没有光致变色性能, 只有无定型膜经过 UV 照射, 可从黄色变成紫色 ${ }^{[10]}$. 2010 年, 山东大学 $\mathrm{Ma}$ 等 ${ }^{[11]}$ 合成了基于 $\mathrm{BTE}$ 和芢二酰亚 胺基础上的一维纳米管, BTE 部分在紫外和可见光的照 射下能进行光致变色反应, 可以应用于数据计算和存 储. 另一方面, 卟啉化合物 ${ }^{[12,13]}$ 良好的光、热稳定性和
光电性能使其在分子开关、分子逻辑门和分子导线等方 面已展现出诱人的应用前景 ${ }^{[14]}$. 本文设计合成了一种 新型的将 BTE 与卟啉化合物键链的光致变色分子, 其 合成路线见 Scheme 1, 并研究了卟啉基团对 BTE 部分 光致变色的影响.

\section{1 实验部分}

\section{1 仪器与试剂}

IR 采用 Bruke Tensor27 红外光谱仪(KBr 压片); NMR 采用 VARIAN INOVA-400 核磁共振仪, TMS 为内 标; MS 采用 Esquire HCT PLUS 型质谱仪; UV-Vis 采用 HITACHI U3010 紫外光谱仪; 元素分析采用 Vario EL

\footnotetext{
*E-mail: zenghp@scnu.edu.cn

Received April 17, 2011; revised November 7, 2011; accepted December 12, 2011.

Project supported by the National Natural Science Foundation of China (No. 21071054), the Science and Technology Planning Project of Guangdong Province (Nos. 2008B010800030, 2009B091300045), the Natural Science Foundation for the Doctoral Program of Guangdong Province (No. 9451064101003131) and the Fundamental Research Funds for the Central Universities (No 2009ZM0179).

国家自然科学基金(No. 21071054)、广东省科技计划(Nos. 2008B010800030，2009B091300045)、广东省自然科学基金博士启动基金(No. 9451064101003131)和中央高校基本科研业务费专项资金(No. 2009ZM0179)资助项目.
} 

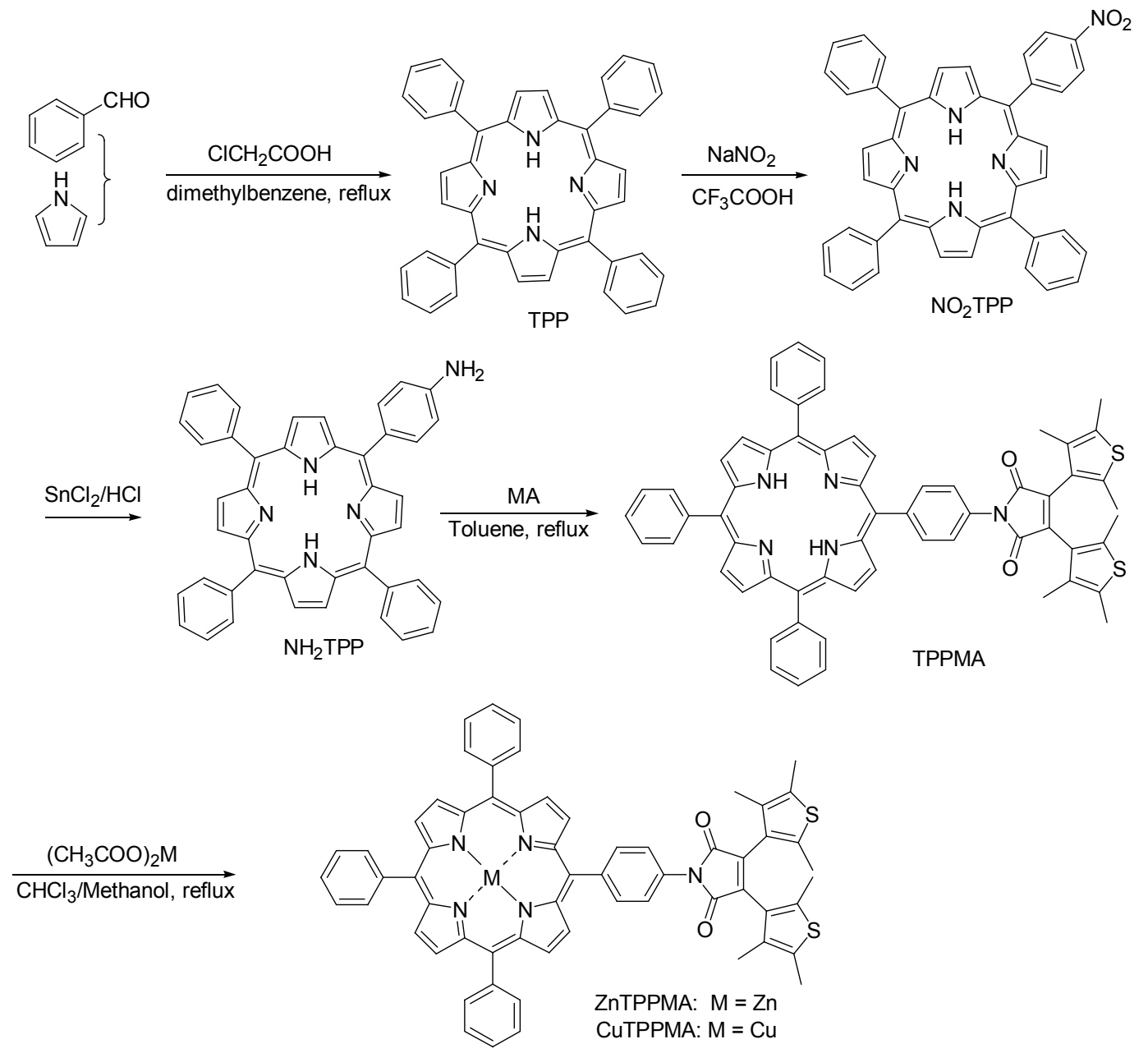

Scheme 1

III 元素分析仪; 苂光光谱采用 HITACHI F-2500 荧光光 谱仪; 光致变色反应光源采用 CHF-XM-500W 短弧氙 灯/录灯稳流电源. 2,3-双(2,4,5-三甲基-3-噻吩)马来酸䣶 购于东京化成(TCI); 吡咯购于上海阿拉丁公司; 氯化 亚锡购于天津市红岩化学试剂厂; 聚乙烯醇缩丁醛树脂 (PVB)购于上海晶纯试剂有限公司; 柱层析和薄层层析 硅胶购自青岛海洋化工厂; 实验所用试剂均为市售分析 纯, 未经纯化直接使用.

\section{2 化合物的合成与表征}

1.2.1 meso-5,10,15,20-四苯基卟啉(TPP)和 5-(4-硝基 苯基)-10,15,20-三苯基卟啉 $\left(\mathrm{NO}_{2} \mathrm{TPP}\right)$ 的合成

参照文献 $[15,16]$ 的方法合成 TPP 和 $\mathrm{NO}_{2} \mathrm{TPP}$.

1.2.2 5-(4-氨基苯基)-10,15,20-三苯基卟啉 $\left(\mathrm{NH}_{2} \mathrm{TPP}\right)$ 的合成

参照文献[17 19]的方法合成 $\mathrm{NH}_{2} \mathrm{TPP}$ : 在装有回 流冷凝管和温度计的 $50 \mathrm{~mL}$ 的三口烧瓶中加入 $20 \mathrm{~mL}$ 浓盐酸和 $0.35 \mathrm{~g} \mathrm{SnCl}_{2} \cdot 2 \mathrm{H}_{2} \mathrm{O}$, 磁力搅拌使其充分溶解,
再加入 $0.20 \mathrm{~g}(0.31 \mathrm{mmol}) \mathrm{NO}_{2} \mathrm{TPP}$. 室温下反应 $30 \mathrm{~min}$ 后, 加热至 $65 \sim 70{ }^{\circ} \mathrm{C}$ 再继续反应 $60 \mathrm{~min}$. 反应混合物 冷却至室温, 加入少量蒸馏水, 析出绿色固体, 过滤, 滤饼依次用饱和食盐水 $(15 \mathrm{~mL})$ 、浓氨水 $(15 \mathrm{~mL})$ 和蒸馏 水 $(10 \mathrm{~mL} \times 3)$ 洗涤后, 干燥得紫黑色固体. 以 $V($ 乙酸乙 酯)： $V$ (石油醚 $)=1: 5$ 为洗脱剂对其进行柱层析分离, 收集第二色带即为还原产物, 干燥后得 $0.17 \mathrm{~g} \mathrm{NH}_{2} \mathrm{TPP}$, 产率 $89 \%, R_{\mathrm{f}}=0.43$ [ $V$ (乙酸乙酯) $: V$ (石油醚) $=1: 3$ ]. UV-Vis $\left(\mathrm{CH}_{2} \mathrm{Cl}_{2}\right) \lambda_{\max }: 416,513,546,593,649 \mathrm{~nm} ;{ }^{1} \mathrm{H}$ NMR (400 MHz, $\left.\mathrm{CDCl}_{3}\right) \delta: 8.93(\mathrm{~d}, J=4.4 \mathrm{~Hz}, 2 \mathrm{H}), 8.83$ (s, 6H), $8.22 \sim 8.20(\mathrm{~m}, 6 \mathrm{H}), 7.98(\mathrm{~d}, J=8.0 \mathrm{~Hz}, 2 \mathrm{H})$, $7.78 \sim 7.71(\mathrm{~m}, 9 \mathrm{H}), 7.01(\mathrm{~d}, J=8.0 \mathrm{~Hz}, 2 \mathrm{H}), 3.94(\mathrm{~s}, 2 \mathrm{H})$, -2.75 (s, 2H); IR (KBr) v: 3455, 3372, 3315, 3053, 3024, 2921, 1734, 1615, 1514, 1474, 1441, 1351, 1282, 980, 800, $702 \mathrm{~cm}^{-1}$; APCI-MS m/z (\%): $629.0\left([\mathrm{M}+1]^{+}, 100\right)$.

1.2.35-[2,3-双(2,4,5-三甲基-3-塞吩基)马来酰亚胺$\mathrm{N}$-苯基]-10,15,20-三苯基卟啉(TPPMA)的合成 参照文献[20]的方法合成 TPPMA: 称取 $0.05 \mathrm{~g} 2,3-$ 
双(2,4,5-三甲基-3-噻吩)马来酸酎 $(0.14 \mathrm{mmol}$ )和 $0.56 \mathrm{~g}$ $\mathrm{NH}_{2}$ TPP $(0.89 \mathrm{mmol})$ 于三口烧瓶中, 加入 $25 \mathrm{~mL}$ 甲苯, $0.2 \mathrm{~mL}$ 三乙胺, $\mathrm{N}_{2}$ 气保护下加热回流, TLC 监控. $24 \mathrm{~h}$ 后停止反应, 冷却至室温, 析出固体, 过滤, 将滤饼用 饱和食盐水 $(10 \mathrm{~mL} \times 3)$ 洗涤, 干燥, 以 $V$ (乙酸乙酯) : $V($ 石油醚 $)=1: 30$ 为洗脱剂对其进行柱层析分离, 收集 第二色带即为 TPPMA, 干燥得紫色固体 $0.11 \mathrm{~g}$, 产率 $83 \%, R_{\mathrm{f}}=0.29$ [ $V$ (乙酸乙酯) $: V($ 二氯甲烷 $)=1: 2$ ] $;{ }^{1} \mathrm{H}$ NMR $\left(400 \mathrm{MHz}, \mathrm{CDCl}_{3}\right) \delta: 8.95$ (d, $\left.J=4.4 \mathrm{~Hz}, 2 \mathrm{H}\right)$, $8.90 \sim 8.87(\mathrm{~m}, 6 \mathrm{H}), 8.27$ (d, $J=8.0 \mathrm{~Hz}, 2 \mathrm{H}), 8.15$ (d, $J=$ $7.6 \mathrm{~Hz}, 6 \mathrm{H}), 7.85(\mathrm{~d}, J=8.4 \mathrm{~Hz}, 2 \mathrm{H}), 7.72 \sim 7.65(\mathrm{~m}, 9 \mathrm{H})$, $2.15(\mathrm{~s}, 6 \mathrm{H}), 2.00(\mathrm{~s}, 6 \mathrm{H}), 1.83(\mathrm{~s}, 6 \mathrm{H}) ;{ }^{13} \mathrm{C}$ NMR $(100$ $\left.\mathrm{MHz}, \mathrm{CDCl}_{3}\right) \delta: 169.3,150.3,142.8,142.0,137.3,137.1$, $136.8,136.5,134.9,134.4,132.1,132.0,131.8,130.7$, $127.5,126.5,123.6,121.2,119.9,29.7,14.0,13.2$; IR (KBr) v: 3313, 2923, 2858, 2360, 2336, 1718, 1620, 1387, 1149, 1091, 965, 800, 735, 699, 658, $600 \mathrm{~cm}^{-1}$; APCI-MS $m / z(\%): 957.7\left(\mathrm{M}^{+}, 100\right)$. Anal. calcd for $\mathrm{C}_{62} \mathrm{H}_{47} \mathrm{~N}_{5} \mathrm{O}_{2} \mathrm{~S}_{2}: \mathrm{C}$ 77.71, H 4.94, N 7.31, S 6.69; found C 77.73, H 4.82, N $7.38, \mathrm{~S} 6.81$.

1.2.45-[2,3-双(2,4,5-三甲基-3-噻吩基)马来酰亚胺$\mathrm{N}$-苯基]-10,15,20-三苯基卟啉锌(ZnTPPMA)和 5-[2,3双 (2,4,5-三甲基-3-噻吩基) 马来酰亚胺- $\mathrm{N}$-苯基]10,15,20-三苯基吓啉铜(CuTPPMA)的合成

参照文献[21]的方法合成 ZnTPPMA 和 CuTPPMA: 在 $100 \mathrm{~mL}$ 的三口烧瓶中加入 $0.05 \mathrm{~g}$ TPPMA $(0.052$ $\mathrm{mmol}$ )和 $20 \mathrm{~mL} \mathrm{CHCl}_{3}, \mathrm{~N}_{2}$ 保护下回流摚拌 $10 \mathrm{~min}$ 后, 通 过恒压滴液漏斗滴加 $10 \mathrm{~mL}$ 溶有 $0.12 \mathrm{~g}$ 醋酸锌 $(0.52$ $\mathrm{mmol}$ )的甲醇溶液, 继续回流搅拌 $2 \mathrm{~h}$. 反应结束后, 将 混合物用蒸馏水洗涤 $(10 \mathrm{~mL} \times 3)$, 少量无水 $\mathrm{Na}_{2} \mathrm{SO}_{4}$ 干 燥过夜, 蒸发除去溶剂. 粗产物以乙酸乙酯/石油醚为洗 脱剂对其进行硅胶柱层析分离.

ZnTPPMA: $0.46 \mathrm{~g}$, 产率 $87 \%, R_{\mathrm{f}}=0.42$ [ $V$ (乙酸乙 酯)： $V$ (石油醚) $=1: 6] ;{ }^{1} \mathrm{H}$ NMR $\left(400 \mathrm{MHz}, \mathrm{CDCl}_{3}\right) \delta$ : $9.02(\mathrm{~d}, J=4.4 \mathrm{~Hz}, 2 \mathrm{H}), 8.96 \sim 8.94(\mathrm{~m}, 6 \mathrm{H}), 8.34(\mathrm{~d}, J=$ $8.4 \mathrm{~Hz}, 2 \mathrm{H}), 8.23 \sim 8.21(\mathrm{~m}, 6 \mathrm{H}), 7.92(\mathrm{~d}, J=8.4 \mathrm{~Hz}, 2 \mathrm{H})$, $7.79 \sim 7.72(\mathrm{~m}, 9 \mathrm{H}), 2.22(\mathrm{~s}, 6 \mathrm{H}), 2.07(\mathrm{~s}, 6 \mathrm{H}), 1.91(\mathrm{~s}$, $6 \mathrm{H}) ;{ }^{13} \mathrm{C}$ NMR $\left(100 \mathrm{MHz}, \mathrm{CDCl}_{3}\right) \delta: 169.5,150.5,143.1$, $142.2,137.6,137.4,137.0,136.7,135.1,134.7,132.3$, $132.2,131.9,131.0,127.7,126.8,123.8,121.4,120.1$, 29.9, 14.6, 13.4; IR (KBr) v: 2964, 2918, 2853, 2363, 2336, 1713, 1385, 1261, 1097, 1022, 800, $700 \mathrm{~cm}^{-1}$; APCI-MS $m / z$ (\%): 1020.5 ([M+1] $\left.]^{+}, 100\right)$. Anal. calcd for $\mathrm{C}_{62} \mathrm{H}_{45} \mathrm{~N}_{5} \mathrm{O}_{2} \mathrm{~S} 2 \mathrm{Zn}$ : C 72.89, H 4.44, N 6.86, S 6.28; found C 72.76, H 4.66, N 6.71, S 6.33.
CuTPPMA: $0.48 \mathrm{~g}$, 产率 $90 \%, R_{\mathrm{f}}=0.59$ [ $V($ 乙酸乙 酯) $： V($ 石油醚 $)=1: 6] ;{ }^{1} \mathrm{H}$ NMR $\left(400 \mathrm{MHz}, \mathrm{CDCl}_{3}\right) \delta$ : $8.99(\mathrm{~d}, J=4.4 \mathrm{~Hz}, 2 \mathrm{H}), 8.91 \sim 8.89(\mathrm{~m}, 6 \mathrm{H}), 8.32$ (d, $J=$ $8.0 \mathrm{~Hz}, 2 \mathrm{H}), 8.20 \sim 8.17(\mathrm{~m}, 6 \mathrm{H}), 7.89(\mathrm{~d}, J=8.8 \mathrm{~Hz}, 2 \mathrm{H})$, $7.75 \sim 7.68(\mathrm{~m}, 9 \mathrm{H}), 2.10(\mathrm{~s}, 6 \mathrm{H}), 1.95(\mathrm{~s}, 6 \mathrm{H}), 1.78(\mathrm{~s}$, $6 \mathrm{H}) ;{ }^{13} \mathrm{C}$ NMR $\left(100 \mathrm{MHz}, \mathrm{CDCl}_{3}\right) \delta: 170.9,168.9,142.4$, $141.6,137.1,136.9,136.6,136.3,135.9,134.6,133.4$, $131.9,131.6,131.2,130.6,127.4,127.2,127.0,126.3$, 123.2, 121.3, 120.1, 29.2, 13.9, 13.0; IR (KBr) v: 2964, 2922, 2850, 2364, 2343, 1714, 1383, 1260, 1095, 1022, 800, $701 \mathrm{~cm}^{-1}$; APCI-MS m/z (\%): 1019.5 ([M+ 1] , 100). Anal. calcd for $\mathrm{C}_{62} \mathrm{H}_{45} \mathrm{~N}_{5} \mathrm{O}_{2} \mathrm{~S}_{2} \mathrm{Cu}: \mathrm{C} 73.03, \mathrm{H} 4.45, \mathrm{~N}$ 6.87, S 6.29; found C 73.25, H 4.54, N 6.68, S 6.33.

\section{2 结果与讨论}

\subsection{TPPMA, CuTPPMA 和 ZnTPPMA 的结构表征}

\subsubsection{TPPMA, CuTPPMA 和 ZnTPPMA 的 IR 谱图}

从 TPPMA, CuTPPMA 和 ZnTPPMA 的红外光谱可 以看出, $3300 \mathrm{~cm}^{-1}$ 左右是卟啉环内 $\mathrm{N}-\mathrm{H}$ 伸缩振动的特 征吸收峰, $965 \mathrm{~cm}^{-1}$ 处为 TPPMA 卟啉环内 $\mathrm{N}-\mathrm{H}$ 的面内 摇摆振动吸收峰. $1715 \mathrm{~cm}^{-1}$ 左右的强吸收峰为 $\mathrm{C}=\mathrm{O}$ 的 伸缩振动. 在与金属配位后, $3300 \mathrm{~cm}^{-1}$ 左右的 $\mathrm{N}-\mathrm{H}$ 伸 缩振动峰和 $965 \mathrm{~cm}^{-1}$ 处的 $\mathrm{N}-\mathrm{H}$ 面内摇摆振动峰消失, 在 $1000 \mathrm{~cm}^{-1}$ 附近出现了新的强吸收峰, 这表明金属离 子已进入卟啉环形成金属配体化合物.

\subsubsection{TPPMA, CuTPPMA 和 ZnTPPMA 的 UV 谱图}

图 1 为化合物 TPPMA, ZnTPPMA 和 CuTPPMA 在 正已烷中的紫外-可见吸收光谱, 浓度均为 $1 \times 10^{-5}$ $\mathrm{mol} / \mathrm{L}$. TPPMA 在 500 700 nm 之间有 4 个吸收峰, 分别 是: $511,545,590,647 \mathrm{~nm}$. 当 TPPMA 中卟啉环与金属 $\mathrm{Cu}$ 和 $\mathrm{Zn}$ 配位后, 吸光度明显减弱，且在 $500 \sim 700 \mathrm{~nm}$ 之间的 $\mathrm{Q}$ 带有变化. CuTPPMA 在 500 700 nm 之间只 有 1 个吸收峰 $(537 \mathrm{~nm})$; ZnTPPMA 在 $545 \mathrm{~nm}$ 处有一较 强的吸收峰, 并在 $597 \mathrm{~nm}$ 处有一小包峰. TPPMA 的 Soret 带在 $415 \mathrm{~nm}$, ZnTPPMA 的 Soret 带在 $415 \mathrm{~nm}$, CuTPPMA 的 Soret 带在 $412 \mathrm{~nm}$, 有稍许蓝移. 这些都是 因为在卟啉环内引入金属离子后, 环内共轭程度增加, 配合物对称性增加, 而使得 $\mathrm{Q}$ 带吸收峰的个数减少、减 弱, Soret 带发生位移 ${ }^{[22]}$.

\subsubsection{TPPMA, CuTPPMA 和 ZnTPPMA 的 FL 谱图}

图 2 是化合物 TPPMA, ZnTPPMA 和 CuTPPMA 在 正已烷 $\left(1 \times 10^{-5} \mathrm{~mol} / \mathrm{L}\right)$ 中的苂光光谱, 激发波长均为 $416 \mathrm{~nm}$. 如图所示, TPPMA 在 $650 \mathrm{~nm}$ 左右有一尖锐的 


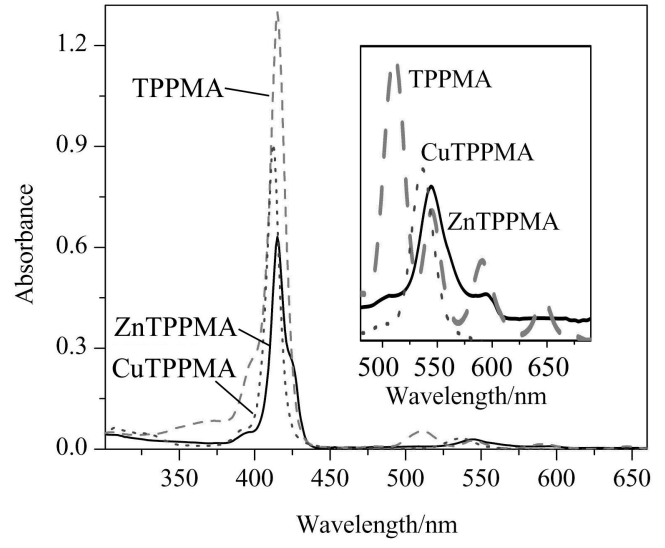

图 1 TPPMA, ZnTPPMA, CuTPPMA 在正己烷中的紫外-可 见吸收光谱

Figure 1 UV-Vis spectra of TPPMA, ZnTPPMA and CuTPPMA in hexane

荧光发射峰, 另在 586 和 $712 \mathrm{~nm}$ 处有小包峰. ZnTPPMA 在 586 和 $633 \mathrm{~nm}$ 处有中等强度发射峰, 在 $549 \mathrm{~nm}$ 处有 一包峰. 由于 CuTPPMA 属于开壳金属卟啉类化合物, 荧光发生淬灭, 所以在正已烷溶液中观察不到 CuTPPMA 的苂光.

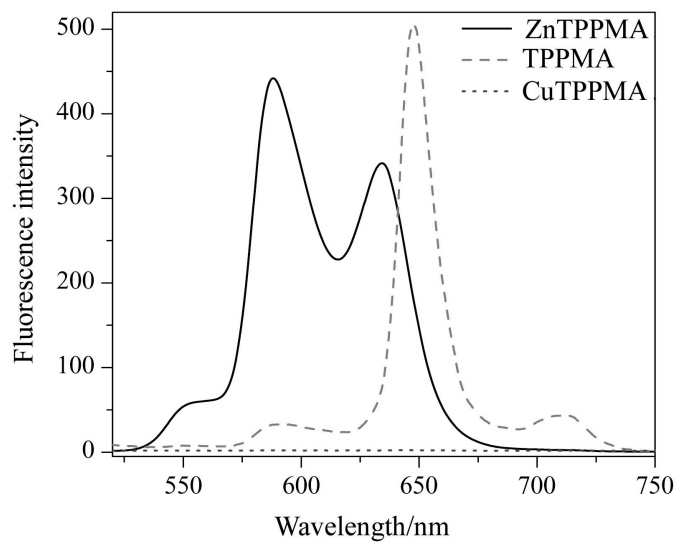

图 2 TPPMA, ZnTPPMA 和 CuTPPMA 在正己烷中的荧光光 谱

Figure 2 Fluorescence spectra of TPPMA, ZnTPPMA and CuTPPMA in hexane

\section{2 光致变色性质}

\section{2 .1 在溶液中的光致变色反应}

将化合物 TPPMA, ZnTPPMA 和 CuTPPMA 分别 溶于正己烷和乙腈中, 配成浓度为 $1 \times 10^{-5} \mathrm{~mol} / \mathrm{L}$ 的 溶液.

图 3 为 TPPMA 的正己烷溶液经 $254 \mathrm{~nm}$ 的紫外 光分别照射 $0,10,20,30,40,50 \mathrm{~s}$ 后的 UV-Vis 光谱, 没
有新的吸收峰出现，吸收峰的强度也没有任何变化. 因此, TPPMA 并没有表现出光致变色性质.

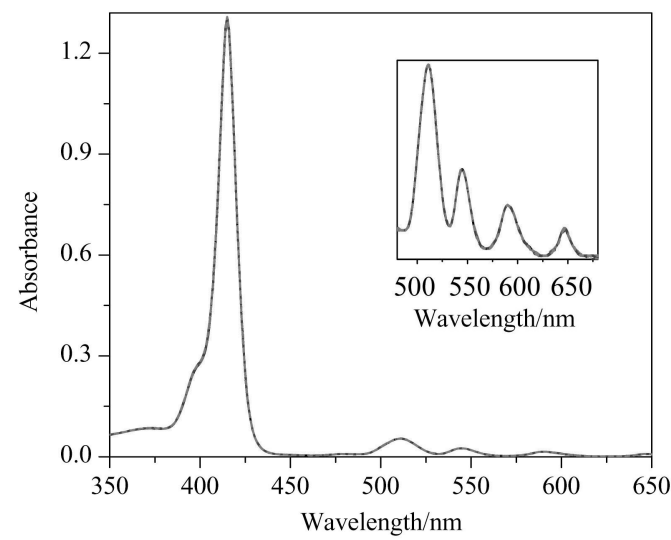

图 $3254 \mathrm{~nm}$ 光照射下, TPPMA 在正己烷中的光谱变化

Figure 3 Absorption spectra of TPPMA in hexane upon irradiation with $254 \mathrm{~nm}$ light

图 4(a)和图 4(b)分别为 ZnTPPMA 在正己烷和乙
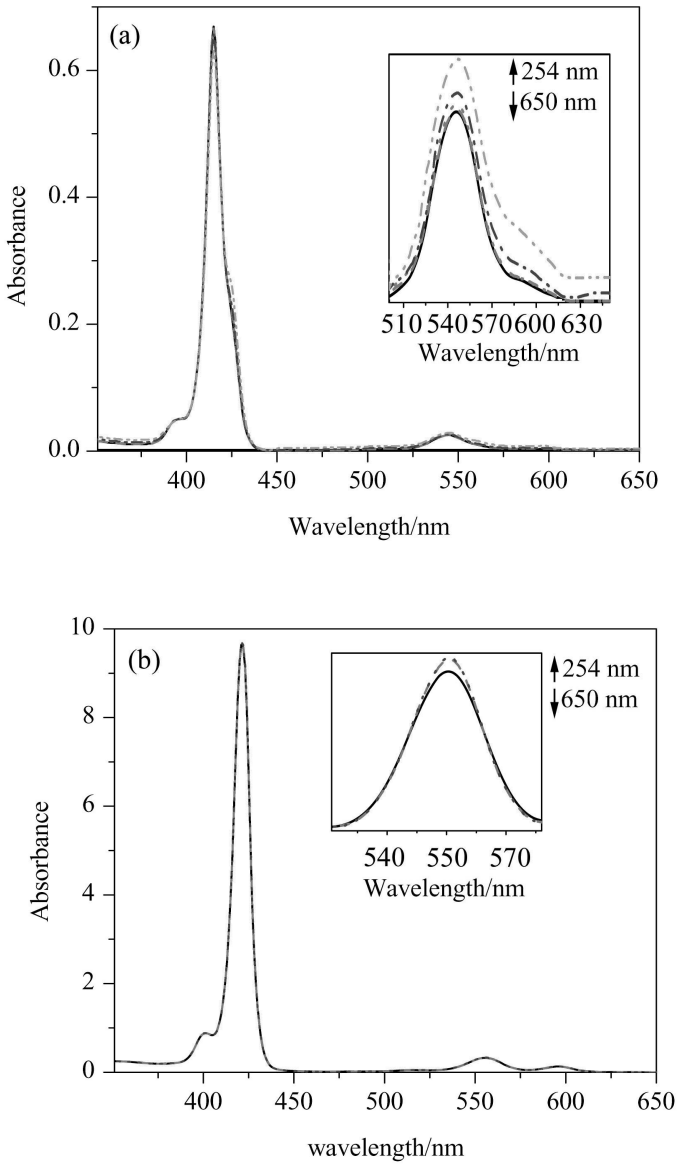

图 4 ZnTPPMA 的正己烷溶液(a)和乙腈溶液(b)经 254 和 650 $\mathrm{nm}$ 光照射后的吸收光谱图

Figure 4 Absorption spectra of ZnTPPMA in hexane (a) and acetonitrile (b) upon irradiation with 254 and $650 \mathrm{~nm}$ light 
腈中经 254 和 $650 \mathrm{~nm}$ 光照射后的紫外-可见吸收光谱. 在图 4(a)中, ZnTPPMA 的正已烷溶液经 $254 \mathrm{~nm}$ 光照后, $546 \mathrm{~nm}$ 处的吸收峰强度逐渐增强，当照射时间达到 $35 \mathrm{~s}$ 后, 吸收强度不再增加, 体系达到光稳定的状态. 此时, 再经 $650 \mathrm{~nm}$ 的可见光照射, $546 \mathrm{~nm}$ 处的吸收峰强度逐 渐降低，当照射时间达到 $50 \mathrm{~min}$ 后，吸收曲线回到起始 状态. 类似地, 在图 4(b)中, ZnTPPMA 的乙腈溶液经 $254 \mathrm{~nm}$ 光照后, $556 \mathrm{~nm}$ 处的吸收峰强度逐渐增强, 当照 射时间达到 $40 \mathrm{~s}$ 后, 吸光度不再增加, 此时, 再经过 650 $\mathrm{nm}$ 的可见光照射, $556 \mathrm{~nm}$ 处的吸光度逐渐降低, 并经过 $50 \mathrm{~min}$ 的照射回到起始状态. 比较图 4(a)和图 4(b)可以 发现, ZnTPPMA 在正己烷中的光致变色性能要强于在 乙腈溶剂中的, 因为对称的二芳基乙烯类化合物的光致 变色反应在极性溶剂中受到极大的抑制 ${ }^{[23]}$.

图 5(a)和图 5(b)分别为 CuTPPMA 的正己烷和乙腈 溶液经 254 和 $650 \mathrm{~nm}$ 光照射后的紫外-可见吸收光谱图. 在图 5(a)中, CuTPPMA 的正己烷溶液经过 $254 \mathrm{~nm}$ 光照 射后, $536 \mathrm{~nm}$ 处的吸收峰强度逐渐增强, 当照射时间达
到 $40 \mathrm{~s}$ 后, 吸收强度不再增加, 体系达到光稳定的状态. 此时再用 $650 \mathrm{~nm}$ 的可见光照射, $536 \mathrm{~nm}$ 处的吸收峰强 度逐渐降低，当照射时间达到 $45 \mathrm{~min}$ 后，吸收曲线回到 起始状态. 类似地, 在图 5(b)中, CuTPPMA 的乙腈溶液 经 $254 \mathrm{~nm}$ 光照后, $537 \mathrm{~nm}$ 处的吸收峰的强度逐渐增强, 当照射时间达到 $60 \mathrm{~s}$ 后，吸光度不再增加，此时再经 $650 \mathrm{~nm}$ 的光照, $537 \mathrm{~nm}$ 处的吸光度逐渐降低, $45 \mathrm{~min}$ 后 曲线回到初始状态. 比较图 5(a) 和图 5(b) 可以发现 CuTPPMA 在正己烷中的光致变色性能也要强于在乙腈 溶剂中的.

\subsection{2 在 PVB 膜中的光致变色反应}

将 $1.0 \mathrm{~g} \mathrm{PVB}, 0.01 \mathrm{~g}$ TPPMA 及 $0.5 \mathrm{~mL}$ 邻苯二甲酸 二丁酯置于 $50 \mathrm{~mL}$ 烧瓶中, 再加入 $10 \mathrm{~mL}$ 乙酸乙酯, 机械 搅拌 $2 \mathrm{~h}$ 后, 静置 $1 \mathrm{~h}$. 采用涂布法将上述混合物均匀涂 布在 $2 \mathrm{~mm}$ 厚的无机玻璃上，室温晾干得到 TPPMA 的 PVB 薄膜. 再照此方法制备浓度为 $1 \%$ 的 CuTPPMA 和 ZnTPPMA 的 PVB 薄膜(图 6).

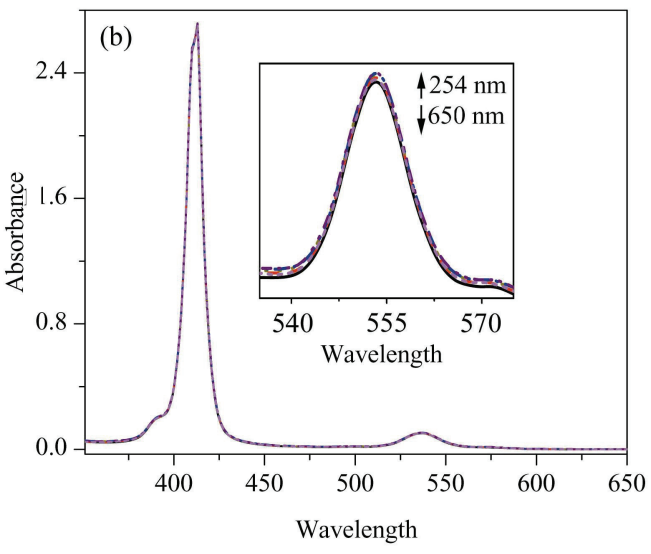

图 5 CuTPPMA 的正己烷溶液(a)和乙腈溶液(b)经 254 和 $650 \mathrm{~nm}$ 光照射后的吸收光谱图

Figure 5 Absorption spectra of CuTPPMA in hexane (a) and acetonitrile (b) upon irradiation with 254 and $650 \mathrm{~nm}$ light
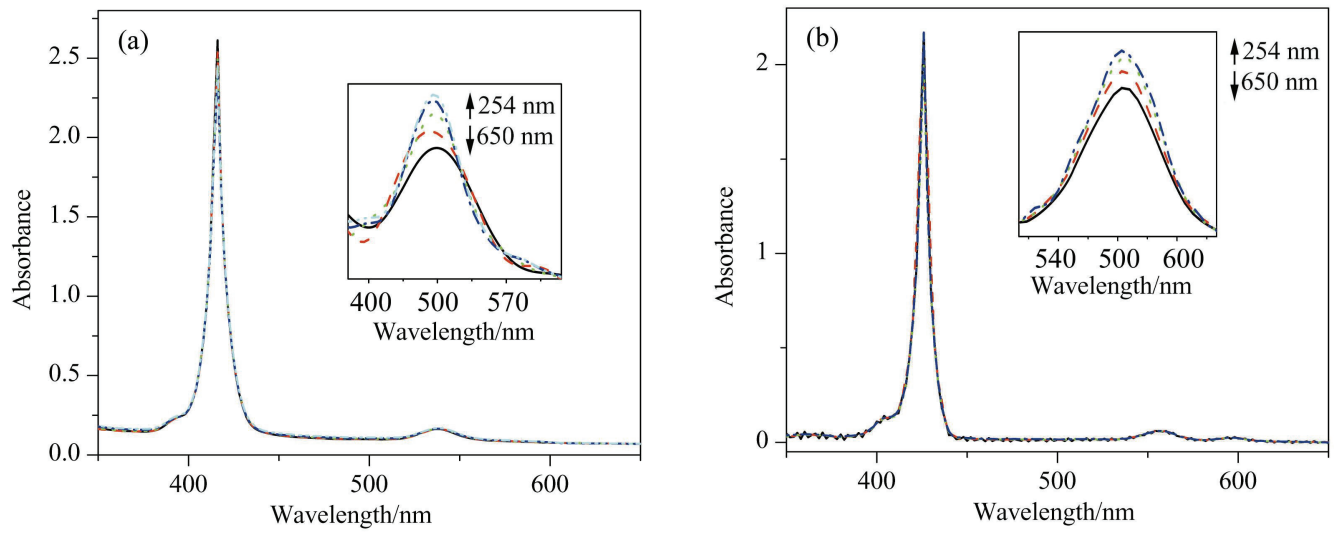

图 6 (a) CuTPPMA/PVB 膜和(b) ZnTPPMA/PVB 膜经 254 和 $650 \mathrm{~nm}$ 光照射后的吸收光谱图

Figure 6 Absorption spectra of CuTPPMA/PVB (a) and CuTPPMA/PVB (b) upon irradiation with 254 and $650 \mathrm{~nm}$ light 
在 PVB 膜中, 仍然没有观测到 TPPMA 的光致变色 现象, CuTPPMA 和 ZnTPPMA 有着与其在正己烷溶液 中完全相似的光致变色特性: 当用 $254 \mathrm{~nm}$ 紫外光照射 时, 546 和 $556 \mathrm{~nm}$ 处的吸收峰分别增强, 对应闭环态的 生成; 若再用 $650 \mathrm{~nm}$ 的可见光照射, 吸收曲线回到起始 状态，闭环态重新回到开环态.

\subsection{3 不同介质对化合物光致变色性能的影响}

通过紫外-可见吸收光谱研究了介质对 CuTPPMA 和 ZnTPPMA 的光致变色反应时间的影响. 图 7(a) 和 7(b)分别表示 ZnTPPMA 和 CuTPPMA 在正己烷、乙腈 及 PVB 薄膜中达到光稳态的时间. 相对于正己烷和乙 腈溶液, 化合物在 PVB 中的光致变色反应时间明显变 长, 这是由于高分子介质的高粘度限制了化合物开环体 的自由关环造成的.

\section{3 荧光性质}

图 8(a)和 8(b)是 ZnTPPMA 的正已烷和乙腈溶液 $\left(1 \times 10^{-5} \mathrm{~mol} / \mathrm{L}\right)$ 经 $254 \mathrm{~nm}$ 光照射前后的苂光光谱. 图
8 (a)中以 $420 \mathrm{~nm}$ 的光作为激发波长，分别在 586 和 633 $\mathrm{nm}$ 处有荧光发射峰. 当用 $254 \mathrm{~nm}$ 的光照射 ZnTPPMA 达光稳态后, 荧光强度明显降低, 荧光峰面积是初始荧 光峰面积的 $88 \%$, 这说明在正已烷中有 $12 \%$ 的 ZnTPPMA 发生了关环反应. 图 8(b)中以 $420 \mathrm{~nm}$ 的光作 为激发波长, $600 \mathrm{~nm}$ 处有一尖锐的荧光发射峰, $649 \mathrm{~nm}$ 处有一小包峰. 当用 $254 \mathrm{~nm}$ 的光照射 ZnTPPMA 达光稳 态后，荧光强度有所降低，苂光峰的面积是初始荧光峰 面积的 $92 \%$, 这说明在乙腈中有 $8 \%$ 的 ZnTPPMA 发生 了关环反应. 由此也可以看出, 在极性溶剂中, 光致变 色反应受到了抑制.

实验结果表明, TPPMA 在 $254 \mathrm{~nm}$ 紫外光照射下, 没有光致变色现象, 而 CuTPPMA 和 ZnTPPMA 在 254 和 $650 \mathrm{~nm}$ 的光照下发生了 Scheme 2 中所示的可逆的开 关环反应, 即光致变色反应. TPPMA 在 $254 \mathrm{~nm}$ 的紫外 光激发下, 发射出 $\lambda=650 \mathrm{~nm}$ 的强烈的荧光, 这有可能 使得已经关环的 TPPMA 再次开环, 导致我们观察不到

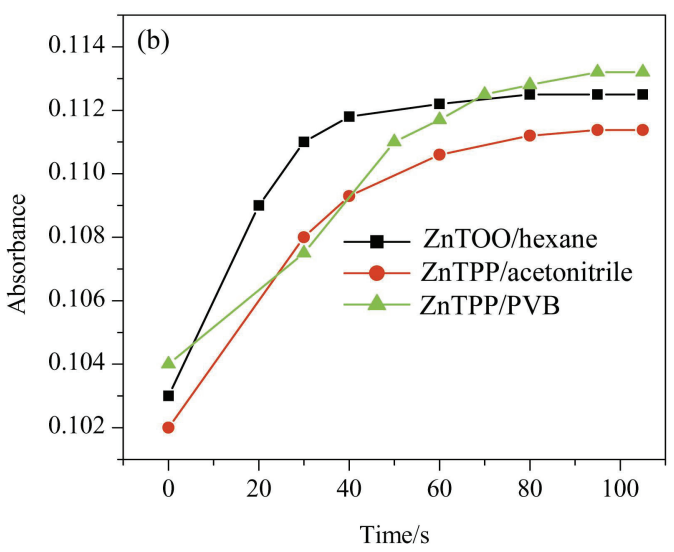

图 7 (a) ZnTPPMA 和(b) CuTPPMA 在不同介质中关环反应时间

Figure 7 Coloration time of (a) ZnTPPMA and (b) CuTPPMA in different media
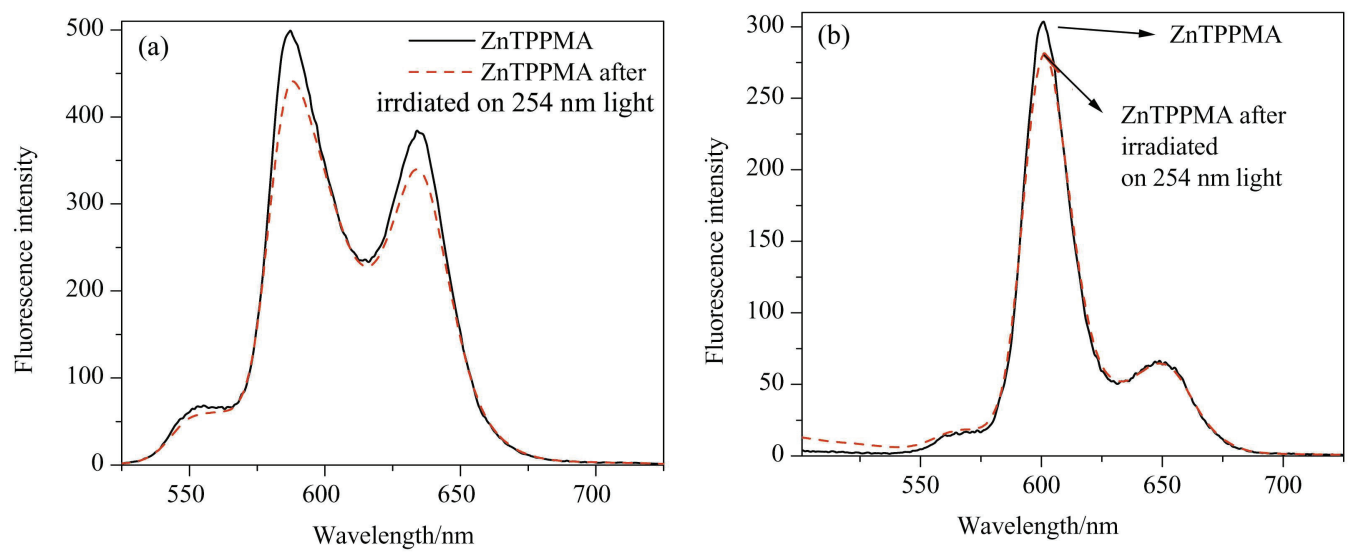

图 $8254 \mathrm{~nm}$ 光照下 ZnTPPMA 在(a)正己烷和(b)乙腈中的荧光光谱

Figure 8 Fluorescence spectra of ZnTPPMA in hexane (a) and acetontrile (b) upon irradiation with $254 \mathrm{~nm}$ light 
TPPMA 光致变色反应在紫外光谱上的变化. 而 ZnTPPMA 荧光峰位置有所变化, 同时强度降低, 而 CuTPPMA 没有苂光, 因此 ZnTPPMA 和 CuTPPMA 都 表现出较明显的光致变色现象.

\section{3 结论}

通过亚胺化反应，合成了新型化合物 TPPMA 及其
金属衍生物 ZnTPPMA 和 CuTPPMA，通过 IR, MS, ${ }^{1} \mathrm{H}$ MR 和 ${ }^{13} \mathrm{C} N M R$ 确认了化合物的结构, 并利用 UV-Vis 光谱探讨了化合物的光致变色性能. 实验结果表明, TPPMA 在 $254 \mathrm{~nm}$ 紫外光照射下, 未能观测到光致变色 现象，而其金属配合物 CuTPPMA 和 ZnTPPMA 在 254 和 $650 \mathrm{~nm}$ 的光照下显示出较好的光致变色性能.
$254 \mathrm{~nm}$<smiles>Cc1sc(C)c(C2=C(c3c(C)sc(C)c3C)C(=O)N(C(C)C)C2=O)c1C</smiles><smiles></smiles>

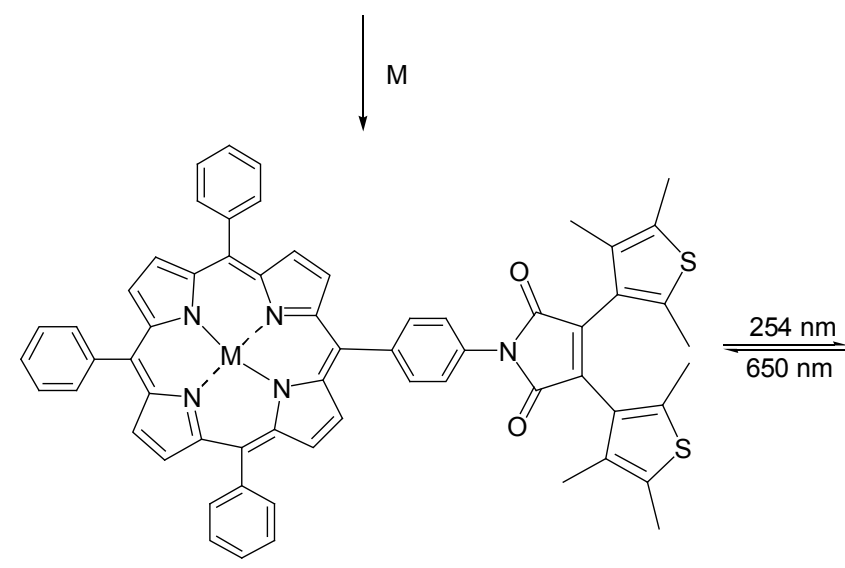

Scheme 2

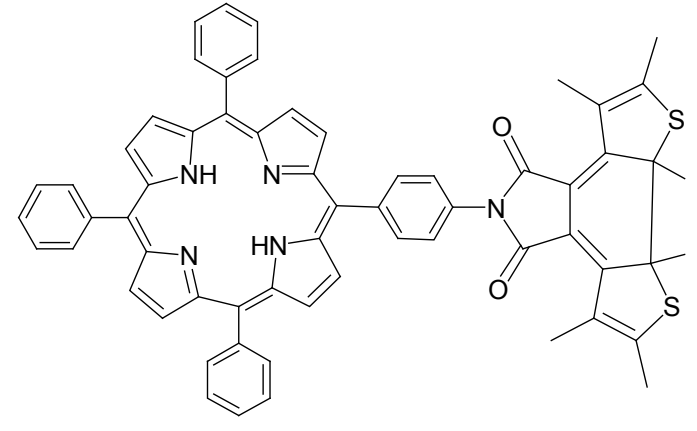
M

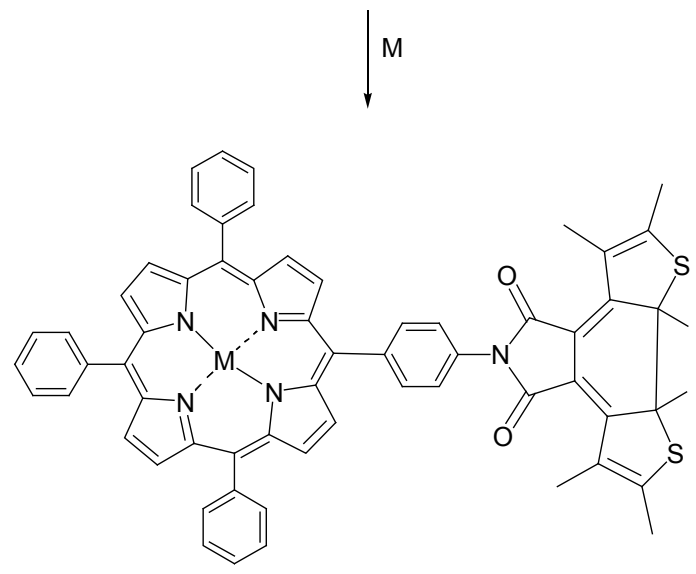

$\mathrm{M}=\mathrm{Cu}$ or $\mathrm{Zn}$

(1)

\section{References}

[1] Aloïse, S.; Sliwa, M.; Pawlowska, Z.; Réhault, J.; Dubois, J.; Poizat, O.; Buntinx, G.; Perrier, A.; Maurel, F.; Yamaguchi, S.; Takeshita, M. J. Am. Chem. Soc. 2010, 132, 7379.

[2] Areephong, J.; Logtenberg, H.; Browne, W. R.; Feringa, B. L. Org. Lett. 2010, 12, 2132.

[3] Zhu, Y.-L.; Zhou, X.-X.; Yin, Q.-F.; Cao, L. Chin. J. Org. Chem. 2009, 29, 34 (in Chinese). (朱玉兰, 周金金金金, 尹起范，曹丽，有机化学, 2009, 29, 34.)

[4] Luo, Q.-F.; Fan, Q.-L.; Huang, W. Chin. J. Org. Chem. 2007, 27, 175 (in Chinese). (罗千福, 范曲立, 黄维, 有机化学, 2007, 27, 175.)

[5] Fukaminato, T.; Doi, T.; Tanaka, M.; Irie, M. J. Phys. Chem. C 2009, 113, 11623.

[6] Zhang, C.; Zhou, H.-P.; Liao, L.-Y.; Feng, W.; Sun, W.; Li, Z.-X.; Xu, C.-H.; Fang, C.-J.; Sun, L.-D.; Zhang, Y.-W.; Yan, C.-H. $A d v$. Mater. 2010, 22, 633.

[7] Patel, P. D.; Masunov, A. E. J. Phys. Chem. A 2009, 113, 8409.

[8] Zheng, H.-Y.; Zhou, W.-D.; Yuan, M.-J.; Yin, X.-D.; Zuo, Z.-C.; Ouyang, C.-B.; Li, Y.-L.; Zhu, D.-B. Tetrahedron Lett. 2009, 50,
1588.

[9] Irie, M.; Mohri, M. J. Org. Chem. 1988, 53, 803.

[10] Kasatani, K.; Kambe, S.; Irie, M. J. Photochem. Photobiol., A: Chem. 1999, 122, 11.

[11] Ma, L.-L.; Wang, Q.-B.; Lu, G.-F.; Chen, R.-P.; Sun, X. Langmuir 2010, 26, 6702 .

[12] Wang, S.-J.; Peng, Y.-L.; Fu, L. Chin. J. Inorg. Chem. 2009, 25, 54 (in Chinese).

(王树军, 彭玉苓, 傅丽, 无机化学学报, 2009, 25, 54.)

[13] Zhang, J.-B.; Li, C.-P.; Huo, T.-R.; Liu, Z.-Y.; Liu, L.-H.; Zhang, T.; Zhang, D.-Y.; Wei, X.-H. Chin. Chem. Lett. 2010, $21,787$.

[14] Zhuang, C.-F.; Zhang, Y.-J.; Xia, A.-Q.; Lian, W.-H.; Wang, Y.-F.; Zhang, P.; Shi, T.-S. Chin. Chem. Lett. 2010, 21, 769.

[15] Yang, X.-G. Ph.D. Dissertation, Zhejiang University, Hangzhou, 2003 (in Chinese). (杨新国, 博士论文, 浙江大学, 杭州, 2003.)

[16] Luguya, R.; Jaquinod, L.; Fronczek, F. R.; Vicente, M. Graca, H.; Smith, K. M. Tetrahedron 2004, 60, 2757.

[17] Shi, W.-M.; Liu, W.-M.; Tao, J.-C. J. Zhengzhou Univ. (Nat. Sci. Ed.) 2005, 37, 78 (in Chinese).

(石伟民, 刘卫敏, 陶京朝, 郑州大学学报 (理学版), 2005, 37, 
78.)

[18] Wang, J.-C.; Zhao, G.-S.; Zheng, Q.-S.; Hou, J.-Q.; Li, J.-S. J. Yunnan Nationalities Univ. 2007, 16, 178 (in Chinese). (王金城, 赵吉寿, 郑启升, 侯俭秋, 李劲松, 云南民族大学学报 (自然科学版), 2007, 16, 178.)

[19] Li, G.-C. M.S. Thesis, Hunan University, Changsha, 2005 (in Chinese).

(李国成, 硕士论文, 湖南大学, 长沙, 2005.)

[20] Fukaminato, T.; Irie, M. Adv. Mater. 2006, 18, 3225.
[21] Liu, B. M.S. Thesis, Northwestern Polythchnical University, Xi'an, 2007 (in Chinese)

(刘波, 硕士论文, 西北工业大学, 西安, 2007.)

[22] Zhang, X.-H.; Jiao, Z.; Yan, W.-W.; Ruan, W.-J.; Zhu, Z.-A. Acta Phys.-Chim. Sin. 2010, 26, 701 (in Chinese).

(张晓红, 矫志, 问伟伟, 阮文娟, 朱志昂, 物理化学学报, 2010, 26, 701.)

[23] Ohsumi, M.; Hazama, M.; Fukaminato, T.; Irie, M. Chem. Commun. 2008, 28, 3281. 